\title{
Efecto de un programa de atención primaria en salud sobre la funcionalidad familiar desde abuelos y nietos, Guapi - Cauca
}

\author{
Effect of a program of primary health attention on family functionality from grandparents and grandchildren in the \\ municipality of Guapi - Cauca
}

Vilma Florisa Velásquez-G1*, Yenny Marcela Barreto-Zorza²

1. Enfermera, MSc en Enfermería. Profesora Asociada. Universidad Nacional de Colombia - Sede Bogotá - Facultad de Enfermería - Departamento Salud de Colectivos- Investigadora, Grupo de Investigación en Cuidado Cultural de la Salud". e-mail: vvelasquez@unal.edu.co orcid.org/0000-0002-5232-9073

2. Enfermera, MSc en Salud Pública. Docente. Escuela de Medicina y Ciencias de la Salud Universidad del Rosario. Participante del Grupo de Investigación Cuidado Cultural de la Salud. Universidad Nacional de Colombia, Sede Bogotá, Colombia. email: ymbarretoz@unal.edu.co orcid.org/0000-0002-5881-0693

Fecha de recepción: Enero 19 - $2016 \quad$ Fecha de revisión: Julio 25 - $2016 \quad$ Fecha de aceptación: Diciembre 12 - 2016

Velásquez-G VF, Barreto Y. Efecto de un programa de atención primaria en salud sobre la funcionalidad familiar desde abuelos y nietos, Guapi - Cauca. Univ. Salud. 2016;18(3):514-524. DoI: http://dx.doi.org/10.22267/rus.161803.56

\section{Resumen}

Objetivo: Determinar el efecto en la funcionalidad familiar de un programa de Atención Primaria en Salud desde abuelos y nietos, en Guapi-Cauca. Materiales y métodos: Estudio cuasi experimental sin grupo control. Participaron 50 familias afrocolombianas. El programa fue construido con la comunidad, con acciones de cuidado adaptadas culturalmente y en entornos saludables. Información recolectada con la Escala de Efectividad del Funcionamiento Familiar (ASF-E 20), procesada y analizada con métodos descriptivos y prueba de rango signado de Wilcoxon. Resultados: El programa de APS obtuvo un efecto estadísticamente significativo en la funcionalidad de las familias desde los nietos (Valor-p < 0,001) y abuelos (Valor-p 0.004). Conclusiones: El programa de APS con estrategia de entornos saludables y cuidados culturalmente congruentes, tiene un efecto en la efectividad de la funcionalidad familiar en los abuelos y nietos de las familias afrocolombianas del Municipio de Guapi Cauca. Favoreció la funcionalidad familiar acciones de promoción de la salud a través de alianzas estratégicas y oportunidades en el desarrollo individual y colectivo de niños y abuelos.

Palabras clave: Atención primaria en salud; relaciones familiares; enfermería en salud comunitaria; niño; adulto mayor. (Fuente: DeCS, Bireme).

\begin{abstract}
Objective: To determine the effect of a Primary Health Attention program on family functionality from grandparents and grandchildren in the municipality of Guapi in the department of Cauca. Materials and methods: A quasiexperimental study without control group was performed where 50 Afro-Colombian families participated. The program was built with the community by using culturally adapted care actions and healthy environments. The information was collected with the scale of Effectiveness of Family Functioning (ASF-20), and it was processed and analyzed with descriptive methods and range test signed of Wilcoxon. Results: The PHC program obtained a statistically significant effect on the functionality of families from grandchildren (Value-p $<0,001$ ) and grandparents (Value-p $<0,004$ ). Conclusions: The PHC program with strategy of healthy environments and culturally congruent
\end{abstract}


care has effect over effectiveness of family functionality in grandparents and grandchildren in Afro-Colombian families in the municipality of Guapi, Cauca. It favored family functionality actions for the promotion of health through strategic alliances and opportunities for individual and collective development of children and grandparents.

Keywords: Primary health care; family relationships; nursing in community health; child; elderly. (Source: DeCS, Bireme).

\section{Introducción}

Los países latinoamericanos presentan una gran diversidad de sistemas de salud en los cuales la Atención Primaria de Salud (APS) tiene un papel fundamental con diversos alcances y formas de organización ${ }^{1}$, por lo que los modelos de atención de salud deben basarse en la APS, orientarse a la familia y la comunidad, incorporar el enfoque de ciclo de vida, tener en cuenta las consideraciones de género y las particularidades culturales, y fomentar el establecimiento de redes de atención de salud y la coordinación social que vela por la continuidad adecuada de la atención ${ }^{2}$.

Desde 1978, la Declaración de Alma Ata refiere que "la atención primaria de salud es la asistencia sanitaria esencial basada en métodos y tecnologías prácticos, científicamente fundados y socialmente aceptables, puesta al alcance de todos los individuos y familias de la comunidad", por tanto representa un contacto directo entre el sistema de salud y las familias y un elemento fundamental para reconocer que la salud de las personas debe ser integral, asegurando la participación activa de las familias y comunidad en la planificación, organización, ejecución y control de la APS 3 .

Por lo anterior, es importante considerar la familia como espacios de socialización, formación, cuidado y protección de sus miembros, especialmente de los más vulnerables: los niños, niñas, adolescentes, adultos mayores y quienes tienen alguna discapacidad. Por consiguiente, el análisis de la problemática de las familias requiere considerar las relaciones que se establecen entre todos sus miembros, puesto que al interior se establecen arreglos específicos para el cuidado y protección de quienes la integran ${ }^{4}$.

Algunos artículos señalan que una de las problemáticas generales a escala mundial en las familias es el envejecimiento poblacional, y el incremento de las enfermedades crónicas no transmisibles, que a largo tiempo puede producir un deterioro funcional en las personas mayores, situación que trae como consecuencia una dependencia en las actividades de la vida diaria, que obliga a las familias a asumir funciones y convertirse en cuidadores informales, afectando su funcionalidad familiar ${ }^{5-8}$.

Al respecto, la Organización Mundial de la Salud (OMS) vislumbra que para el año 2025, la población adulta mayor se duplicará, lo que plantea hoy retos al Estado y a la sociedad en su conjunto para atender las crecientes demandas de este grupo poblacional, que debe prever cómo refuerza sus sistemas de protección social para estar en capacidad de atenderlas adecuadamente ${ }^{4}$. Desde lo social, el fenómeno del envejecimiento muestra en América Latina y el Caribe dos características preocupantes: Un ritmo más acelerado históricamente en países en vías de desarrollo y un contexto caracterizado por una persistente desigualdad entre los recursos de la población y un débil progreso institucional, sobre todo en los sistemas de protección social de baja cobertura y calidad, acompañado de una sobrecarga peligrosa para la familia que, junto con asumir las necesidades de las personas de edad avanzada, se compromete con los vacíos derivados de la exigua presencia de las instituciones sociales como dispositivos de protección y cohesión ${ }^{9}$.

Por otra parte, programas realizados con familias con niños y adolescentes reportan la necesidad de reivindicar los hijos en la familia ${ }^{10}$. Hay estudios que indican que los niños y adolescentes presentan diversas conductas de riesgo en la familia, responsables de la morbi-mortalidad en los menores, entre los cuales se destacan: el consumo de drogas, embarazo precoz, infecciones de transmisión sexual, que implican un deterioro 
considerable en la salud, afectando la calidad de vida, la inserción en la sociedad y gran parte de las enfermedades crónicas no transmisibles del adulto $^{11}$.

Un estudio plantea que la falta de apoyo de los servicios sociales y de salud a las familias, además de falta de autocuidado en la familia con personas que padecen enfermedades crónicas y niños que requieren de cuidado, se convierten en una carga que amenaza el bienestar y la calidad de vida de las familias, dado el desequilibrio de la salud y las finanzas de la familia entera y repercute en la funcionalidad familiar ${ }^{12}$.

El hecho de aproximarse a las familias analizando su funcionalidad permite comprender la relación recíproca que se establece entre las familias y sus miembros, donde la salud también tiene una relación significativa con la organización y actitudes familiares e influye en la respuesta de la familia a los cambios ${ }^{13}$. La mayoría de la atención de salud ocurre en el ámbito familiar y si bien la familia ha perdido y transformado muchas de sus funciones, continúa asumiendo un peso importante en el cuidado de la salud, particularmente en la atención al adulto mayor y los niños, donde "se ha desempeñado como institución proveedora de protección social, particularmente activa en los países en desarrollo, por su creciente exposición a oscilaciones macroeconómicas, a riesgos emergentes en un contexto de débil protección pública y pobreza extendida"14. Así, la familia persiste como un pilar fundamental en el régimen de bienestar latinoamericano ${ }^{15}$ aunque no siempre de manera afortunada por la precariedad de recursos para cubrir las necesidades de todos y cada uno de sus miembros ${ }^{16}$.

Los colombianos reconocen que las familias son una fuente clave de apoyo ante la incertidumbre; para el 53\% de ellos la unión familiar y la presencia de la familia extensa es el producto de la necesidad de supervivencia y de la tradición ${ }^{17}$. En las familias extensas existe algo así como "un alto grado de cooperación" que "en casos de emergencia" genera un fuerte sentimiento de solidaridad que aprieta las ramas de parentesco. Por encima de los conflictos entre sus miembros, "salva a esta molécula social de su desintegración el fuerte funcionalismo que para la sobrevivencia individual éste representa". Al fin y al cabo, "son más las razones para permanecer unidos que para disociarse"18. Lo que sugiere que las familias extensas para los abuelos generan más bienestar $^{19}$ en su dinámica interna en la vida cotidiana y que sus miembros se sienten más satisfechos y saludables, posiblemente porque las relaciones dentro de la familia son más intensas que en cualquier grupo de la sociedad ${ }^{20}$, sienten a la familia como fuerte proveedora de actuaciones de bienestar, que brinda apoyo en el plano emotivo, estimulando el sentido de pertenencia y la estima personal ${ }^{21}$, donde ve el cuidado de la familia incorporado de manera agradable, se siente satisfecho y establece una relación positiva con la familia como cuidadora, mantiene una balance positivo en lo que da y recibe de ella21.

Algunos estudios de funcionalidad familiar reportan predominio de familias funcionales en familias con adultos mayores ${ }^{22-24}$, baja y alta en familias con niños preescolares ${ }^{25,26}$, intermedia en familias con niños escolarizados ${ }^{27}$, baja, intermedia y alta funcionalidad en familias con adolescentes ${ }^{28-30}$. No se encontró estudios de familias afrocolombianas que midan la funcionalidad familiar y aplicación de programas.

Las familias afrocolombianas enfrentan condiciones de extrema pobreza y exclusión, las cuales deben ser reconocidas y analizadas como un punto de partida para adoptar las acciones que contribuyan a cerrar las brechas existentes ${ }^{31}$, dado que esta población constituye alrededor del $10,6 \%$ de los colombianos ${ }^{32}$ y por lo general los adultos mayores asumen el rol de la crianza y orientación de los miembros de la familia especialmente de los niños, sin embargo, en algunas circunstancias, ha cambiado el rol de la persona mayor en el hogar, pasando este de ser sabio, respetado y educador, a ser desplazado y olvidado por medios de comunicación, personas que conforman su familia o vecinos o la sociedad en general ${ }^{33}$.

El municipio de Guapi está ubicado en el Cauca (Sur de Colombia) cuenta con una extensión de $2.688 \mathrm{~km}^{2} 34$. De acuerdo con los grupos etarios, en 
este municipio tanto para el año 2005 como para 2013, el 49,6 \% del total de la población, está formada por personas de 0-17 años, de estas el rango con mayor cantidad es el comprendido en las edades de 6-11 años $(17,3 \%)^{32}$. Según proyecciones del DANE la esperanza de vida es de 65 años para los hombres y de 71 años para las mujeres, para un promedio de 68 años ${ }^{35}$.

Las familias afrocolombianas guapireñas en su mayoría son extensas, en ellas el adulto mayor es respetado y valorado por sus conocimientos ancestrales, a través de ellos persisten "sabedores" como los remedieros, curanderos, parteros y sobanderos. Al interior de la mayoría de las familias no hay un trabajo estable y por tanto su situación económica no es muy buena, la mayoría de individuos de una familia desempeña trabajos informales. Por esto, para las personas que habitan este municipio, la familia es el eje central que posee herramientas necesarias para el desarrollo de las personas y la comunidad ${ }^{36}$. Por lo anterior el presente estudio buscó determinar el efecto en la funcionalidad familiar de un programa de Atención Primaria en Salud (APS) desde los nietos y abuelos en Guapi-Cauca.

\section{Materiales y métodos}

Se realizó un estudio cuantitativo, de tipo cuasiexperimental sin grupo control, en el área urbana de Guapi-Cauca, durante siete semanas, con 50 familias extensas, con los siguientes criterios de inclusión: Pertenecer a familias extensas afrocolombianas pobres, con abuelos mayores de 60 años independientes que puedan realizar actividades de la vida diaria y salir de la casa, nietos entre 7 y 12 años escolarizados y sus madres, permanencia de la familia en el municipio de Guapi mayor a 1 año y deseos de participar voluntariamente en el estudio.

El programa de APS fue construido con la comunidad, con acciones de cuidado adaptadas culturalmente, aplicado en entornos saludables, con los abuelos en la iglesia, centro de atención del adulto mayor y el parque y con los nietos en la escuela. Consta de tres proyectos realizados independientemente con nietos, madres $\mathrm{y}$ abuelos, por sugerencia de las familias, con 6 encuentros en cada proyecto, cumplidos con intervalo de ocho días y con una duración promedio de 2 horas por encuentro. Fue aplicado por una enfermera estudiante de maestría en salud pública y experta en geronto-geriatría y enfermería transcultural y dos líderes de la comunidad capacitadas en el programa. Este este artículo presenta los resultados de la aplicación del programa en dos generaciones: abuelos y nietos.

La aplicación del programa de APS, "Centro de Desarrollo Humano Comunitario: Con fuerza, alegría y amor echamos pa'lante las familias", comenzó con una visita a la familia, (denominado encuentro cero), que consistió en la presentación general del programa a las familias, obteniendo en esta reunión la firma del consentimiento informado por parte de abuelos y madres y asentimiento por los nietos y la citación de los participantes al primer encuentro de abuelos o nietos respectivamente.

En el programa para niños se trabajaron temas como: rescate de tradiciones culturales, pautas de crianza, valores, cuidado del cuerpo, desarrollo de habilidades, y cuidado del agua. Por otro lado, el programa para adultos mayores funcionales enfatizó en temas como: hábitos de vida saludable, significado de ser adulto mayor, transmisión de saberes populares, salud ambiental-reciclaje, enfermedad crónica no transmisible-hipertensión arterial, promoción de la salud-bailoterapia, unión familiar $y$ agradecimiento a $\operatorname{Dios}^{36}$.

La información recolectada al iniciar y al finalizar la implementación del programa de APS (variable independiente) midió la efectividad del Funcionamiento Familiar (variable dependiente) con la Escala de Efectividad del Funcionamiento Familiar (ASF-E 20) ${ }^{37}$. Esta escala es útil para una evaluación de la salud familiar y para comprender las cuatro metas (estabilidad, espiritualidad, control y crecimiento). Los miembros de la familia señalan afirmaciones de algunos aspectos de la funcionalidad familiar, clasificadas de acuerdo con un nivel de efectividad de la familia, el puntaje máximo es de 60 puntos y el mínimo es de 20 
puntos, clasificados así: Bajo: 20 -50 puntos; intermedio 51- 55 puntos; y alto 56-60 puntos. El instrumento presenta validez de constructo, de contenido y concurrente con un coeficiente del alfa de Cronbach de $0.60-0.84^{37}$. De acuerdo con la doctora Friedemann, autora de la escala, son fiables los datos globales y las metas, por tanto, aquí se presentan estos resultados.

Se codificó y elaboró dos bases de datos de las familias correspondientes a nietos y abuelos. Posteriormente, se procesó esta información en el paquete estadístico SPSS, versión 19. Como guía de análisis se utilizaron los datos de caracterización de las familias con miembros de cada familia, edad, sexo, tipología familiar, ocupación, situación económica, así como la funcionalidad familiar general y metas familiares, incluidos en el ASF-ES 20, verificándose la existencia de diferencias estadísticamente significativas para las dos muestras independientes (nietos y abuelos) con la prueba de rango signado de Wilcoxon.

Las hipótesis planteadas fueron:

Hipótesis nula. El Programa de Atención Primaria no tiene efecto sobre la funcionalidad familiar en los abuelos y nietos de las familias afrocolombianas del municipio de Guapi Cauca.

Hipótesis alterna. El Programa de Atención Primaria tiene efecto sobre la efectividad de la funcionalidad familiar en los abuelos y nietos de las familias afrocolombianas del municipio de Guapi Cauca.

\section{Aspectos éticos}

Se solicitó autorización a la autora del instrumento (ML Friedemann) y a la Alcaldía del municipio de Guapi. El estudio contó con aval del Comité de Ética de Investigación de la Facultad de Enfermería, además se presentó en su totalidad a la comunidad y a las instituciones involucradas, con la investigación, posteriormente se resolvieron inquietudes, se informó que la participación era voluntaria y se podían retirar en el momento que consideraran pertinente; se solicitó la firma del consentimiento y asentimiento informado, asegurando que la decisión de participar fuera voluntaria y que no afectara la relación terapéutica u otros beneficios a que tenían derecho los participantes y al finalizar el programa quienes requirieron de apoyo de servicios de salud fueron remitidos a la respectiva entidad de salud. Fueron considerados todos los aspectos éticos para un estudio de bajo riesgo de acuerdo con la resolución 8430 de 1993 del Ministerio de Salud de Colombia.

\section{Resultados}

Las 50 familias participantes, están conformadas por 233 personas, la población femenina supera a los hombres (63\%), hay un promedio de 5 personas por familia, cerca de la mitad de la población son niñas, niños y adolescentes (40\%), y de estos, el $21.9 \%$ son menores entre 7 y 12 años. El grupo de 19-59 años representa el 33\% del total de la población y $27 \%$ son adultos mayores de 60 años. Los 50 nietos participantes, en su totalidad son nacidos en Guapi-Cauca, el $52 \%$ son de sexo femenino y todos se encuentran entre 7-12 años de edad y están escolarizados. Los 50 abuelos participantes, son adultos mayores funcionales, la mayoría nacidos en Guapi (82\%), y de otros municipios cercanos pertenecientes a la zona del Pacífico colombiano, apoyan económicamente a su familia (68\%), en gran parte reciben entre $\$ 50.000$ y $\$ 100.000$ mensuales $(45 \%)$ proveniente principalmente de un subsidio (42\%), seguidos por la ayuda de la familia (32\%) y trabajo informal/actividad propia $(16 \%)$.

\section{Efecto del programa de APS en la funcionalidad familiar}

A nivel general, se destaca que tanto nietos como abuelos estiman de manera similar la baja funcionalidad de sus familias y mostraron cambios de la funcionalidad familiar después de aplicado el programa de APS, disminuyendo el nivel bajo (88\% en la preprueba a $66 \%$ en la posprueba), y aumentando el nivel intermedio de la efectividad de la funcionalidad familiar (pasó de 12 a 34\%) (Tabla 1).

Llama la atención que ningún nieto y abuelo considera su familia en un nivel alto de funcionalidad, posiblemente por las condiciones de pobreza y exclusión, a pesar de que los abuelos 
viven en la familia con sus hijas y nietos, muchos tienden a sentir soledad, por ello algunas veces consideran que su familia no los quiere o simplemente los rechaza, manifestado con frases como: "...cuando uno empieza a dejar de funcionar lo empiezan a dejar a un lado..." (YR); "...como uno ya no trabaja entonces se siente como un estorbo" (EP); “...todo el día en la casa sin tener con quien hablar uno se amarga...” (AO).

Tabla 1. Puntaje Global de la Evaluación de la Efectividad de la Funcionalidad Familiar en los nietos escolares y abuelos funcionales en las familias extensas en Guapi 2014

\begin{tabular}{|c|c|c|c|c|c|c|c|c|}
\hline \multirow{3}{*}{$\begin{array}{l}\text { Nivel de } \\
\text { efectividad } \\
\text { funcionalidad } \\
\text { familiar }\end{array}$} & \multicolumn{4}{|c|}{ Nietos escolares } & \multicolumn{4}{|c|}{ Abuelos funcionales } \\
\hline & \multicolumn{2}{|c|}{ Pre-prueba } & \multicolumn{2}{|c|}{ Post-prueba } & \multicolumn{2}{|c|}{ Pre-prueba } & \multicolumn{2}{|c|}{ Post-prueba } \\
\hline & Frec & $\%$ & Frec & $\%$ & Frec & $\%$ & Frec & $\%$ \\
\hline Bajo & 44 & 88 & 33 & 66 & 44 & 88 & 33 & 66 \\
\hline Intermedio & 6 & 12 & 17 & 34 & 6 & 12 & 17 & 34 \\
\hline Alto & 0 & 0 & 0 & 0 & 0 & 0 & 0 & 0 \\
\hline Total & 50 & 100 & 50 & 100 & 50 & 100 & 50 & 100 \\
\hline
\end{tabular}

Figura 1. Distribución porcentual del efecto logrado con la aplicación del programa de APS en la efectividad en la funcionalidad familiar según las cuatro metas familiares desde los nietos

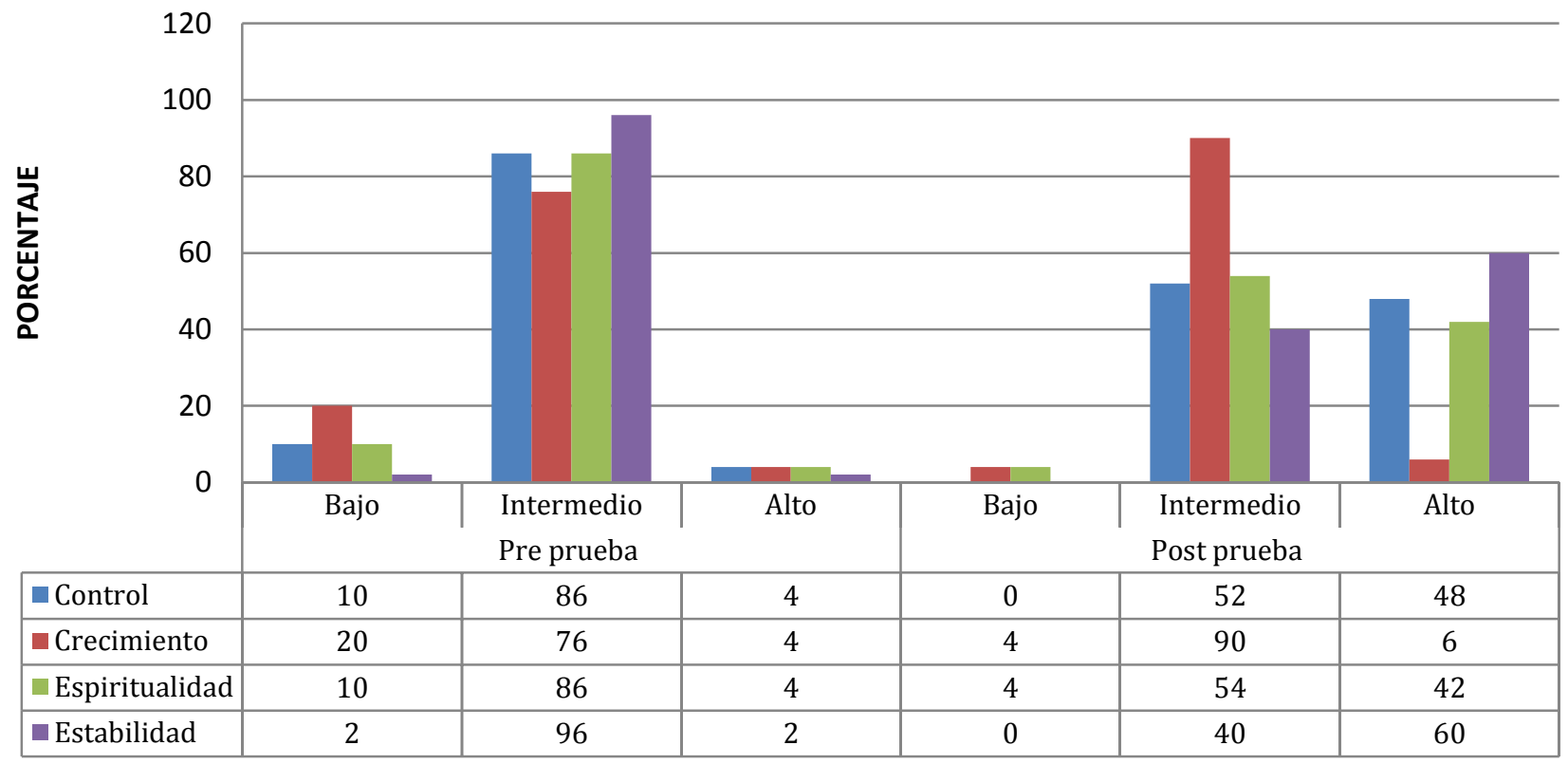

Sin embargo, después de aplicar el programa, más de la mitad de los nietos y abuelos continúan considerando baja la efectividad de la funcionalidad familiar (66\%), con la probabilidad de que las familias que se encuentran en un nivel intermedio vuelvan a migrar a un nivel bajo si las familias no continúan trabajando en las diferentes metas del sistema.

De acuerdo con la prueba de rango signado de Wilcoxon, los resultados presentados tienen un valor-p de cero $(<0.001)$ lo que permite aceptar la 
hipótesis alterna que considera: la aplicación del programa "Centro de Desarrollo Humano Comunitario", tiene efecto sobre la funcionalidad familiar en los nietos (Valor-p $<0,001$ ) y abuelos (valor-p 0.004) de las familias afrocolombianas del Municipio de Guapi Cauca.

Efecto del programa de APS en las metas del sistema familiar desde la percepción de los nietos $y$ adultos mayores

La figura 1 muestra el efecto positivo del programa en todas las metas familiares desde los nietos, evidenciados en la diferencia entre el pre y posprueba, especialmente en el logro alcanzado en la meta de estabilidad alta (pasó de $2 \%$ a $60 \%$ ), control alto (pasó de $4 \%$ a $48 \%$ ) y espiritualidad alta (pasó de $4 \%$ a $42 \%$ ).

De igual forma, la figura 2, muestra el efecto logrado por el programa en la efectividad de la funcionalidad familiar desde los abuelos, especialmente en el control alto (pasó de $17 \%$ a $54 \%$ ), la estabilidad alta (pasó de $38 \%$ a $64 \%$ ) y la espiritualidad alta (pasó de $32 \%$ a $54 \%$ ).

Figura 2. Distribución porcentual del efecto logrado con la aplicación del programa de APS en la efectividad en la funcionalidad familiar según las cuatro metas familiares desde los abuelos

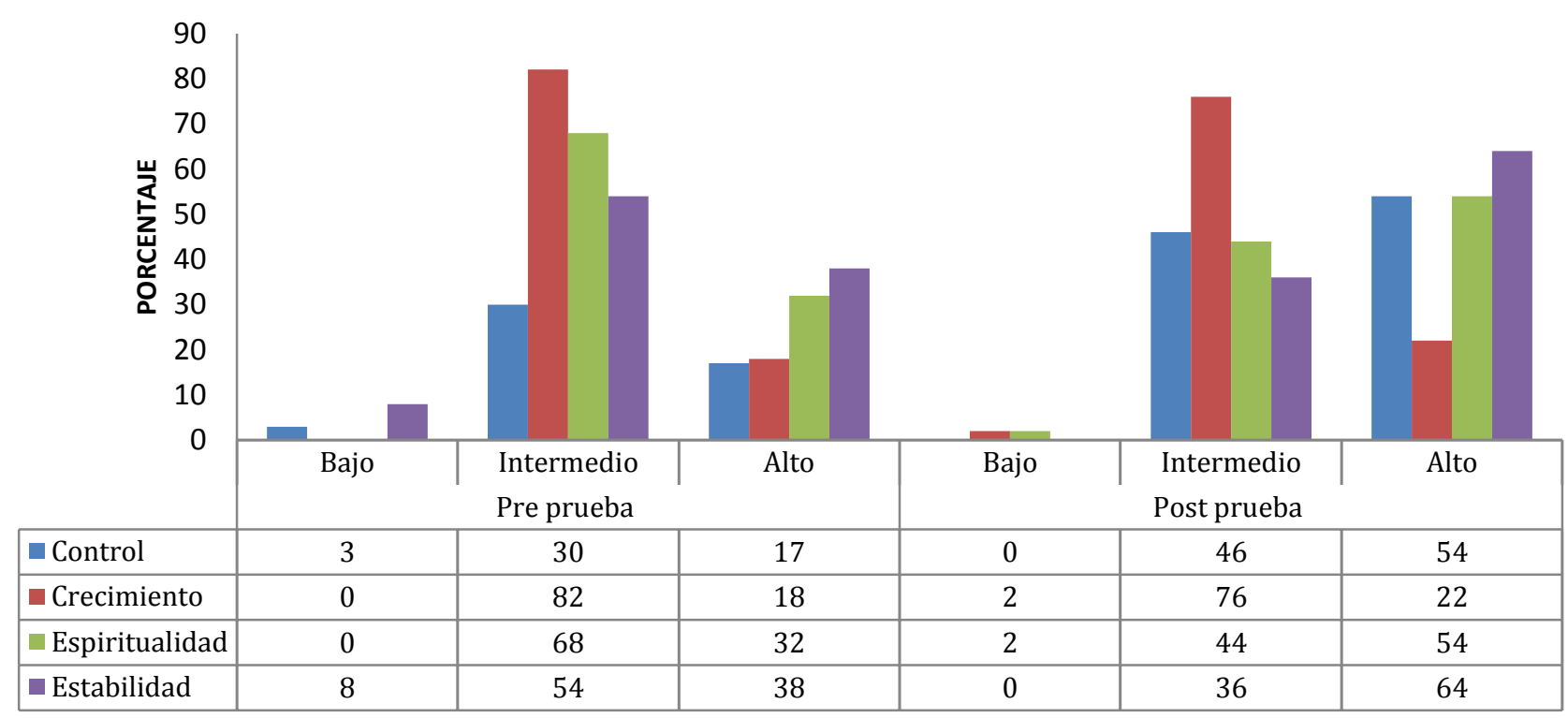

Tabla 2. Evaluación de las metas familiares según nietos y abuelos de las familias extensas en Guapi

\begin{tabular}{lrrrr}
\hline \multicolumn{1}{c}{$\begin{array}{c}\text { Metas de la funcionalidad } \\
\text { familiar/Valor } \mathbf{p}\end{array}$} & \multicolumn{1}{c}{ Estabilidad } & \multicolumn{1}{c}{ Crecimiento } & \multicolumn{1}{c}{ Control } & \multicolumn{1}{c}{ Espiritualidad } \\
\hline Nietos & $<0,001$ & 0,001 & $<0,001$ & $<0,001$ \\
Abuelos & $<0,001$ & 0,981 & 0,016 & 0,003 \\
\hline
\end{tabular}

Por tanto, la tabla 2 evidencia que la aplicación del programa de APS tiene un efecto estadísticamente significativo desde los nietos y los abuelos en la efectividad de las metas de funcionalidad familiar en las familias afrocolombianas del municipio de Guapi Cauca.

\section{Discusión}

Algunas investigaciones centradas en familias con adultos mayores realizadas en Colombia, muestran predominio de familias extensas, extensa modificada y atípica ${ }^{22,38-41}$, y destaca la 
satisfacción de las personas mayores por sus familias ${ }^{40-42}$.

Algunos estudios de funcionalidad familiar de familias con adultos mayores muestran predominio de familias funcionales ${ }^{23-25}$ coincidiendo parcialmente con lo reportado por Ortega et al.43, a la funcionalidad familiar de los adultos mayores estudiadas y contrario con lo expresado por sus familiares, que su mayoría calificó las familias disfuncionales. Es importante resaltar que los resultados respaldan la afirmación de la CEPAL, que la pobreza afecta más intensamente a hogares extensos y compuestos ${ }^{44}$ $\mathrm{y}$ a familias altamente vulnerables como las afrocolombianas, que en el presente estudio reflejan baja efectividad de la funcionalidad familiar en los nietos y adultos mayores, afectada principalmente por la meta crecimiento, cuando la familia no logra establecer procesos efectivos en el cambio del sistema, donde se exponen dificultades para dar respuestas a las necesidades de cada uno de sus miembros, de aceptar o rechazar nuevos valores que facilitan el crecimiento familiar.

Los resultados de baja funcionalidad familiar en familias extensas afrocolombianas presentados en este documento son afines a los hallazgos en estudios de familias con niños ${ }^{26-31,45}$ y contrario a resultados de estudios con adultos mayores colombianos que muestran predominio de familias funcionales ${ }^{23-25}$, posiblemente por las condiciones de extrema pobreza y exclusión de las familias participantes en este estudio, las cuales deben ser reconocidas y analizadas como un punto de partida para adoptar acciones que contribuyan a cerrar las brechas existentes ${ }^{42}$.

Los documentos analizados evidencian que para estudiar la familia es necesario abordar los fenómenos que se dan en la estructura familiar y analizar sus funciones dentro del contexto y cultura para comprender los cuidados realizados por esta, las situaciones de riesgo o los problemas de salud. Esto implica que la relación familia y cultura encierra una variedad de cuidados populares para mantener o recuperar la salud y a la vez, contiene factores de riesgo para sus miembros, y es a través de ese reconocimiento que los profesionales de la salud pueden lograr el acercamiento a las familias de diversas culturas ${ }^{46}$. La literatura muestra que el estudio de la relación de familia y cultura es limitado y las investigaciones cualitativas han empezado a descubrir las maneras de cuidado popular más coherentes con la cultura, con el propósito de promover el cuidado de la salud de la familia, donde se favorezca la conexión entre el saber profesional y el saber popular ${ }^{46}$, para lograr efectos en la funcionalidad familiar, como los encontrados en el presente estudio, pero que requieren seguimiento por parte de instituciones del Estado que garanticen protección y crecimiento de las familias.

A pesar de todos los potenciales beneficios para las familias, hasta ahora ha habido un escaso desarrollo de intervenciones dirigidas específicamente a ellas para potenciar sus fortalezas y mejorar su salud. Se necesita mayor investigación para entender cómo los factores psicosociales y familiares afectan el desarrollo de las conductas de salud y conocer los componentes críticos que debieran incluir intervenciones dirigidas a la familia. Es indispensable diseñar, implementar y evaluar innovaciones dirigidas a la promoción del bienestar familiar fortaleciendo sus recursos internos. Así, aumentando el apoyo familiar, mejorando la calidad de relaciones y generando cambios en los hábitos de vida familiar se pudiera contribuir a controlar el avance de las enfermedades crónicas, mejorar la salud de las personas y aumentar la calidad de vida de todos, y la funcionalidad familiar ${ }^{47}$. De ahí que es de vital importancia la familia para las personas, porque vivir en familias saludables se asocia a vivir más, tener una mejor salud y mayor calidad de vida, ésta pudiera ser una unidad en la cual centrar esfuerzos de trabajo clínico y de investigación en la Atención Primaria ${ }^{47}$.

Así mismo, se corrobora como válida la condición inicial de que un programa de APS con enfoque cultural permite a los participantes en las sesiones de trabajo ser escuchados por los otros asistentes, compartir experiencias, valorar la importancia de la familia y compartir con sus familias el aprendizaje logrado en el programa. Como lo propone la literatura, con la creación de 
entornos saludables, donde se espera: "configurar una participación transformadora que evoque y que logre el empoderamiento de las comunidades, una perspectiva política, que rescate los espacios de encuentro entre hombres y mujeres, niños, jóvenes y adultos; que permita el diálogo permanente, sincero y constructivo con los entes territoriales, una participación auténtica en rescate de los valores culturales y sociales de cada comunidad, que respete y dignifique la diversidad y que desde todo punto de vista, logre aunar esfuerzos, sin escatimar el más mínimo de ellos, para lograr el desarrollo humano y social de las familias y comunidades" 48 , aunque actividades como la bailoterapia ejecutadas a campo abierto como el parque, puede sesgar parte de los resultados, a pesar de que culturalmente estas actividades relacionadas con música y baile son más aceptadas a campo abierto que en espacios cerrados por la población afrocolombiana.

De esta manera, el éxito de la Promoción de la Salud radica en "la participación efectiva y concreta de la comunidad en la fijación de prioridades, la toma de decisiones y la elaboración y puesta en marcha de estrategias de planificación para alcanzar un mejor nivel de salud"49, para que el estado de salud de las familias sea más favorable en términos de oportunidades para el desarrollo individual y colectivo de los diversos grupos que integran la sociedad.

Por lo anterior, se considera de suma importancia la necesidad de efectuar investigaciones que generen estrategias, programas y políticas basadas en la aplicación de resultados pertinentes $^{4}$ y de la experiencia vivida por la comunidad, que "requiere que los sistemas de salud pongan a las personas en el centro de la salud sanitaria" 50 , garantizando que su opinión y sus elecciones influyan decisivamente en la manera en que son diseñados y funcionan los servicios de salud ${ }^{51}$. Así, el programa de atención primaria en salud: Centro de Desarrollo Humano Comunitario, contribuye al mejoramiento de las condiciones de vida, funcionamiento familiar y salud de las familias y población afrocolombiana de Guapi.

\section{Conclusiones}

Las familias extensas afrocolombianas pobres residentes en Guapi presentan bajo funcionamiento familiar desde los abuelos y nietos y la aplicación de un programa de APS con enfoque cultural y de entornos saludables, mostró efectos estadísticamente significativos de la funcionalidad familiar en estos dos grupos generacionales, que son un aporte al conocimiento de la funcionalidad familiar y de la salud pública.

\section{Limitaciones}

Dadas las condiciones específicas de la población y el tamaño de la muestra, el estudio no permite hacer inferencias a partir de esta población. Además, a pesar de que los criterios de inclusión garantizan una cierta homogeneidad entre los participantes con respecto a su situación de salud, hay variaciones en sus características internas que pueden influir en su adherencia y respuesta a la intervención. Es importante dejar registro de actividades realizadas especialmente a campo abierto como el baile que pueden generar sesgos en la evaluación del programa.

\section{Financiación}

Universidad Nacional de Colombia, División Investigación Bogotá (DIB). Proyecto: Efecto de un programa de atención primaria en salud para familias extensas afrocolombianas desde un enfoque cultural en el municipio de Guapi-Cauca. Código: 19062.

\section{Agradecimientos}

Las autoras agradecen a la Alcaldía de Guapi, a la Fundación Franciscana Da Amor FFUNDAMOR, a la comunidad del Municipio de Guapi-Cauca, a la Universidad Nacional de Colombia y a las familias que participaron de forma comprometida $y$ desinteresada.

Conflicto de interés: Los autores declaran no tener conflicto de interés. 


\section{Referencias}

1. Organización Panamericana de la Salud. Perfiles básicos de salud de países en las Américas. Argentina, 2004. Washington, D.C.: OPS; 2005.

2. Macinko J, Montenegro H, Nebot Adell C, Etienne C y Grupo de Trabajo de Atención Primaria de Salud de la Organización Panamericana de la Salud. La renovación de la atención primaria de salud en las Américas. Rev Panam Salud Pública. 2007;21(2/3):73-84.

3. Declaración de Alma-Ata. Conferencia Internacional sobre Atención Primaria de Salud, Alma-Ata, URSS. 1978. Disponible en http://www.promocion.salud.gob.mx/dgps/descargas 1/promocion/1_declaracion_deALMA_ATA.pdf

4. Perú. Ministerio de la Mujer y Poblaciones Vulnerables. Boletín Infofamilias, Un espacio para la reflexión. Personas adultas mayores y sus familias. 2012 Sept; 1(3):3-6.

5. Marim C, Silva V, Taminato M, Barbosa DA. Effectiveness of educational programs on reducing the burden of caregivers of elderly individuals with dementia: a systematic review. Rev. Latino-Am. Enfermagem. 2013 Jan.-Feb.; 21 (Spec):267-75.

6. Oliveira DC, D'Elboux MJ. Estudos nacionais sobre cuidadores familiares de idosos: revisão integrativa. Rev Bras Enferm, Brasília 2012 set-out; 65(5): 829-38.

7. Galicia Aguilar RM. Cuidado de enfermería familiar en una comunidad rural. Rev Paraninfo Digital, 2009; 6. [en línea] [Consultado 15 de noviembre de 2015] Disponible en http://www.indexf.com/para/n6/o015.php

8. Alarcón-Cerón M. Modelo integrador de enfermería: una estrategia para la Atención Primaria a la Salud. Rev Enferm Inst Mex Seguro Soc 2013; 21 (1): 35-40.

9. Huenchuan S. Envejecimiento, derechos humanos y políticas públicas, Chile: Cepal; 2009. Disponible en: http://.imsersomayo-

res.csic.es/documentos/documentos/cepalenvejecimiento-05.pdf.

10. Pérez R. El programa de las familias, colección FEAPS. España. [en línea] [Consultado 5 de octubre de 2015] Disponible en: http://www.feaps.org/biblioteca/familias_ydi/capitulo 3.pdf

11. Valenzuela M, Ibarra A, Zubarew T, Correa L. Prevención de conductas de riesgo en el adolescente: rol de familia. Rev. Index de Enfermería [Index Enferm] (edición digital) 2013; 22(1-2). [en línea] [Consultado 5 de mayo de 2014] Disponible en http://www.index-f.com/indexenfermeria/v22n1-2/7942.php

12. Hisako S, Bragança M, Ferraz V, Monteiroi H. Dinâmica da família no contexto dos cuidados a adultos na quarta idade. Rev Bras Enferm, Brasília. 2013 mar-abr; 66(2):228-33.

13. La protección social en América Latina y el Caribe. (Internet). Disponible en: http://.imsersomayores.csic.es/documentos/documen tos/cepal-envejecimiento-01.pdf
14. Gómez E, Muñoz MM, Haz AM. Familias Multiproblemáticas y en riesgo social: características e intervención. Psykhe. 2007; 16 (2): 43-54.

15. Sunkel G. El papel de la familia en la protección social en América Latina, preparado como apoyo al documento. Santiago de Chile: CEPAL; 2006. Disponible en: http://www.eclac.org/publicaciones/DesarrolloSocial/ 0/LCL2530PE/sps120_LCL2530.pdf

16. Amaya-Rey MCP. Informe técnico: Estandarización de instrumentos de Salud Familiar y Riesgo familiar total para Iberoamérica 2005-2012 DIB. Universidad Nacional de Colombia. Facultad de Enfermería: Programa de Doctorado; Bogotá: 2009.

17. Zamudio Cárdenas L, Rubiano BN. Las familias de hoy en Colombia. Bogotá: Formato Comunicación Diseño; 1994: 73.

18. Gutiérrez de Pineda V. Estructura, función y cambio de la familia en Colombia. Bogotá: ASCOFAME; 1975. Disponible http://es.scribd.com/doc/59360954/Gutierrez-dePineda-Virginia-Familia-y-cultura-en-Co-lombia

19. Montoro R, García S, Gamir L, Becker G. Dimensiones económicas y sociales de la familia. Madrid: Fundación Argentaria; 2000.

20. Amaya de Peña P. Riesgo familiar total 7-70 y del ISFGEN 25: referentes teóricos-conceptuales y análisis de la información. Bogotá: Universidad Nacional de Colombia. 2000.

21. Velásquez G VF, Amaya Rey MCP. Riesgo Familiar Total en familias con personas mayores, municipio de Funza (Cundinamarca, Colombia). Salud Uninorte, 2012; 28(2).

22. Zavala-Rodríguez MR, Ríos-Guerra MC, García-Madrid G, Rodríguez-Hernández, CP. Funcionalidad familiar y ansiedad en pacientes adultos con enfermedad crónica. Aquichan. 2009;9(3):257-270.

23. Zavala G, Vidal G, Castro S, Quiroga P, Klassen P. Funcionamiento social del adulto mayor. Cienc. Enferm. 2006; XII (2): 53-62.

24. García G, Landeros E, Arrioja G, Pérez AM. Funcionalidad familiar y capacidades de los adultos mayores en una comunidad rural. Rev Enferm IMSS 2007; 15 (1):21-26.

25. Villalobos $V$ BE. efectividad de la funcionalidad familiar en las familias con hijos en preescolar, matriculados en el colegio San Rafael. [Trabajo de grado, magíster en Enfermería con énfasis en Salud Familiar]. Universidad Nacional de Colombia, Facultad de Enfermería, Bogotá; 2008.

26. Valle R. Funcionalidad familiar de familias con niños preescolares estudiantes de colegios de Bogotá D.C. 2011. Rev colomb enferm. 2012 ago; 7:68-74.

27. Bolaño Castro D, Cortina Guerrero J, Vargas Cerro S. Funcionalidad familiar en niños con dificultades en el aprendizaje escolar en dos instituciones educativas públicas del municipio de Sincelejo. Revisalud Unisucre 2013; 1(1):3.

28. García Rueda A, Martínez de Acosta CH. Efectividad de la funcionalidad familiar en familias con adolescentes gestantes y adolescentes no gestantes. Av. Enferm. 2011; 29 (1):75-86. 
29. Galán-González EF. Funcionalidad en familias con adolescentes escolarizados en Paipa. Av.Enferm. 2010; 28 (1):72-82.

30. Bolívar Y. Efectividad de la funcionalidad familiar en las familias con adolescentes escolarizados. [Trabajo de grado, magíster en Enfermería con énfasis en Salud Familiar], Universidad Nacional de Colombia, Facultad de Enfermería, Bogotá; 2006.

31. PNUD. Programa de las Naciones Unidas para el Desarrollo. Los afrocolombianos frente a los objetivos de desarrollo del milenio. Disponible en http://www.undp.org/content/dam/colombia/docs/0 DM/undp-co-odmafrocolombianos-2012.pdf

32. Departamento Administrativo Nacional de Estadística DANE. 2005. [Internet] Disponible en http://www.dane.gov.co. Consultado [12/12/2015].

33. López Cárdenas WI, Puentes Vacca JS, Soto Mora BH. Necesidades en el contexto de familias afrocolombianas con ancianos municipio Guapi Cauca 2009. [Tesis pregrado en Enfermería]. Universidad Nacional de Colombia. Bogotá: 2009. Disponible en: http://www.bdigital.unal.edu.co/8556/

34. Alcaldía de Guapi-Cauca. Unidos por el cambio. Municipio de Guapi y su administración. 2013. [en línea]. [Consultado 18 de septiembre de 2013]. Disponible en http://guapi-cauca.gov.co/index.shtml

35. Colombia. Ministerio de Trabajo. Perfil productivo Municipio Guapi. Insumo para el diseño y alternativas para la generación de empleo a las víctimas de la violencia. RED ORMET 2013. P 1-132. [en línea]. [Consultado 25 de marzo de 2014]. Disponible en www.mintrabajo.gov.co/component/.../1607perfilproductivoguapi.html

36. Barreto Y. Efecto de un programa de atención primaria en salud para familias extensas afrocolombianas, GuapiCauca, 2014. [Tesis de Maestría en Salud Pública]. Universidad Nacional de Colombia.

37. Friedemann ML. Instrumento de aplicación escala de evaluación de la funcionalidad familiar, versión revisada. Bogotá: Grupo de Investigación Funcionalidad Familiar, Universidad Nacional de Colombia, Facultad de Enfermería; 2008. pp. 1-3.

38. Herrera LM. Caracterización de las familias que conviven con adultos mayores en la ciudad de Villavicencio [Tesis de magíster]. Bogotá: Universidad Nacional de Colombia; 2001.

39. Cataño N. Familias con personas mayores de 60 años y más, que asiste al Club Azucenas del Quindío [Tesis de magíster]. Bogotá: Universidad Nacional de Colombia; 2003.

40. Velásquez GVF. Caracterización de las familias con adultos mayores con discapacidad, Patio Bonito, Bogotá, 2005. Avanc Enferm. 2009; 27(1):69-81.

41. Velásquez VCY, Velásquez GVF. Caracterización de las familias con adultos mayores funcionales, sector Patio Bonito, Bogotá, 2005. Avanc Enferm. 2007;25(2):10-21.

42. Velásquez-G VF, Amaya Rey MCP. Salud familiar en familias con personas mayores en Funza, Cundinamarca. Investig Enferm. Imagen Desarro. 2011;13(2):11-26.

43. Ortega A, Fernández V, Osorio P. Funcionamiento familiar. $\mathrm{Su}$ percepción por diferentes grupos generacionales y signos de alarma. Rev Cubana Med Gen Integr 2003; 19 (4).

44. CEPAL. Pobreza en América Latina: Nuevos escenarios y desafíos de políticas para el hábitat urbano. División de Medio Ambiente y Asentamientos Humanos Santiago de Chile, octubre de 2000. [en línea]. [Consultado 15 de septiembre de 2012]. Disponible en http://www.eclac.org/publicaciones/xml/7/5637/lcl1 429e.pdf

45. Osorno-Navarro MM. Funcionalidad en las familias de las madres comunitarias de los hogares de bienestar infantil de Sincelejo, Colombia. Revista Curare. 2014; $1(1)$.

46. Mejía LD, López L. La familia y la cultura: Una conexión innovadora para el cuidado de la salud. Index Enferm. [revista en la Internet]. 2010;19(2-3):138-142. [consultado 10 de septiembre de 2015] Disponible en: http://scielo.isciii.es/scielo.php?script=sci_arttext\&pid $=$ S1132-12962010000200015\&lng=es.

47. García-Huidobro D. Enfoque familiar en la atención primaria: Una propuesta para mejorar la salud de todos. Rev. méd. Chile 2010; 138 (11): 1463-1464.

48. Dirección Seccional de Salud y Protección Social de Antioquia. Gobernación de Antioquia Lineamientos para la promoción de la salud con-sentido humano en Antioquia. 2010. ISBN: 978-958-8711-00-3

49. Organización Mundial de la Salud. La Carta de Ottawa para la promoción de la salud. 1986. [Consultado 21 de abril de 2014]. Disponible en: http://www.paho.org/spanish/hpp/ottawachartersp.p df.

50. Organización Mundial de la Salud. Informe sobre salud en el mundo 2008. La atención primaria en salud, Más necesaria que nunca. Disponible en http://www.who.int/whr/2008/08_report_es.pdf. Consultado [03/04/2014]

51. Moran M. Governing the health care state: a comparative study of the United Kingdom, The United States and Germany. Manchester and New York NY, Manchester University Press, 1999. 